\title{
Heparin-induced thrombocytopenia during extracorporeal life support: incidence, management and outcomes
}

\author{
Jae Hwan Choi ${ }^{1}$, Jessica G. Y. Luc ${ }^{2}$, Matthew P. Weber ${ }^{1}$, Haritha G. Reddy ${ }^{1}$, Elizabeth J. Maynes ${ }^{1}$, \\ Avijit K. Deb ${ }^{3}$, Louis E. Samuels ${ }^{1}$, Rohinton J. Morris ${ }^{1}$, H. Todd Massey ${ }^{1}$, Antonio Loforte ${ }^{4}$, \\ Vakhtang Tchantchaleishvili ${ }^{1}$
}

${ }^{1}$ Division of Cardiac Surgery, Thomas Jefferson University, Philadelphia, PA, USA; ${ }^{2}$ Division of Cardiovascular Surgery, Department of Surgery, University of British Columbia, Vancouver, British Columbia, Canada; ${ }^{3}$ Philadelphia College of Osteopathic Medicine, Philadelphia, PA, USA; ${ }^{4}$ Department of Cardiovascular Surgery and Transplantation, S. Orsola-Malpighi Hospital, University of Bologna, Bologna, Italy Correspondence to: Vakhtang Tchantchaleishvili, MD. Assistant Professor of Surgery, Division of Cardiothoracic Surgery, Thomas Jefferson University, 1025 Walnut St, Suite 607, Philadelphia, PA 19107, USA. Email: Vakhtang.Tchantchaleishvili@jefferson.edu.

Background: Heparin-induced thrombocytopenia (HIT) is a severe antibody-mediated reaction leading to transient prothrombosis. However, its incidence in patients on extracorporeal life support (ECLS) is not well described. The aim of this systematic review was to report the incidence of HIT in patients on ECLS, as well as compare the characteristics and outcomes of HIT in patients undergoing veno-arterial extracorporeal membrane oxygenation (VA-ECMO) and veno-venous ECMO (VV-ECMO).

Methods: An electronic search was performed to identify all studies in the English literature examining outcomes of patients with HIT on ECLS. All identified articles were systematically assessed using specific inclusion and exclusion criteria. Random effects meta-analysis as well as univariate analysis was performed.

Results: Of 309 patients from six retrospective studies undergoing ECLS, 83\% were suspected, and $17 \%$ were confirmed to have HIT. Due to the sparsity of relevant retrospective data regarding patients with confirmed HIT on ECLS, patient-based data was subsequently collected on 28 patients from case reports and case series. Out of these 28 patients, $53.6 \%$ and $46.4 \%$ of them underwent VA-ECMO and VVECMO, respectively. Patients on VA-ECMO had a lower median platelet count nadir (VA-ECMO: 26.0 vs. VV-ECMO: 45.0 per $\mu \mathrm{L}, \mathrm{P}=0.012$ ) and were more likely to experience arterial thromboembolism (VAECMO: $53.3 \%$ vs. VV-ECMO: 0.0\%, $\mathrm{P}=0.007)$, though there was a trend towards decreased likelihood of experiencing ECLS circuit oxygenator thromboembolism (VA-ECMO: 0.0\% vs. VV-ECMO: 30.8\%, $\mathrm{P}=0.075$ ) and thromboembolism necessitating ECLS device or circuit exchange (VA-ECMO: $13.3 \%$ vs. VV-ECMO 53.8\%, $\mathrm{P}=0.060$ ). Kaplan-Meier survival plots including time from ECLS initiation reveal no significant differences in survival in patients supported on VA-ECMO as compared to VV-ECMO $(\mathrm{P}=0.300)$. Conclusions: Patients who develop HIT on VA-ECMO are more likely to experience more severe thrombocytopenia and arterial thromboembolism than those on VV-ECMO. Further research in this area and development of standardized protocols for the monitoring, diagnosis and management of HIT in patients on ECLS support are warranted.

Keywords: Extracorporeal life support (ECLS); extracorporeal membrane oxygenation (ECMO); heparininduced thrombocytopenia (HIT); thrombosis

Submitted Oct 02, 2018. Accepted for publication Nov 30, 2018.

doi: $10.21037 /$ acs.2018.12.02

View this article at: http://dx.doi.org/10.21037/acs.2018.12.02 


\section{Introduction}

Extracorporeal life support (ECLS) is a form of temporary life support for patients with life-threatening cardiac and/ or respiratory failure that has been increasingly used in recent years with improvements in bypass techniques and devices (1). Veno-venous extracorporeal membrane oxygenation (VV-ECMO) provides respiratory support, while veno-arterial ECMO (VA-ECMO) provides cardio-respiratory support to patients with severe, but potentially reversible, cardiac or respiratory failure refractory to standard therapeutic modalities (2). Bleeding and thrombotic complications remain a leading cause of morbidity and mortality in patients on ECLS (1).

Heparin-induced thrombocytopenia (HIT) is a severe and potentially life-threatening antibody-mediated reaction caused by circulating heparin-platelet factor 4 (H-PF4) complex antibodies leading to a transient prothrombotic condition (3). Thromboembolic complications of HIT can include pulmonary embolism, ischemic limb necrosis necessitating limb amputation, acute myocardial infarction and stroke (4). As exposure of patients on ECLS to unfractionated heparin is universal, the incidence and complications of HIT are important to consider in this population of patients.

The 2012 American College of Chest Physicians HIT guidelines do not provide guidance for the diagnosis and management of HIT in patients on ECLS support (4), likely due to the paucity of clinical evidence in this population. Thrombocytopenia during ECLS support is often what drives clinical suspicion of HIT, though diagnosis of HIT in patients on ECLS support is often confounded by factors inherent to the ECLS circuits themselves and a lack of standardized protocols (1).

The aim of this review is to estimate the incidence of HIT in patients on ECLS support and to compare the characteristics and outcomes of HIT in patients with VAECMO and VV-ECMO.

\section{Methods}

\section{Literature search strategy}

Thorough electronic searches were performed in September 2018 using Ovid Medline, Cochrane Central Register of Controlled Trials (CCTR), Cochrane Database of Systematic Reviews (CDSR), Scopus and CINAHL. Maximum sensitivity of the search strategy was achieved by using the following search terms: (extracorporeal circulation OR ECMO OR ECLS OR assisted circulation OR circulatory assist) AND (thrombocytopenia OR serotonin release assay OR platelet factor OR aggregation). The reference lists of all retrieved articles were reviewed for further identification of potentially relevant studies and assessed using the inclusion and exclusion criteria.

\section{Eligibility criteria}

Eligible studies for the present systematic review and metaanalysis included patients who developed HIT during ECLS support. Patients undergoing cardiopulmonary bypass $(\mathrm{CPB})$ were excluded. Patients $<16$ years of age were also excluded. When institutions published duplicate studies with overlapping individual patient data, only the most complete reports were included for quantitative assessment at each time interval. Due to paucity of data, individual case reports were included in the analysis. We excluded studies not published in the English language and those not involving human subjects.

\section{Definitions}

Suspicion of HIT was raised in patients on ECLS by various factors including a decrease in platelet count by $>50 \%(5-9)$, thrombotic event(s) $(6,9,10)$, change in postoperative status (6), duration of ECLS support $>72$ hours (11) and intermediate to high 4Ts score $(>3)(6,12)$. The cutoff criterion for HIT suspicion was not always specified or uniform for every institution. H-PF4 antibody enzyme-linked immunosorbent assay (ELISA) was performed as a screening test for HIT $(13,14)$, where optical density (OD) values of equal to or greater than 0.4 was considered positive. Following a positive screening test, the diagnosis was usually confirmed by either functional assays, such as serotonin release assay (SRA) $(6,8,15-17)$ or heparin induced platelet aggregation assay (HIPA) $(5,18,19)$, or a strong clinical suspicion of HIT with subsequent recovery of thrombocytopenia following heparin discontinuation $(8,10,20-24)$. High positive ELISA test result (e.g., OD >1.0) alone was sometimes considered sufficient to confirm the diagnosis of HIT $(6,25)$. Pre-nadir platelet count was defined as highest measured platelet count at any time between initiation of ECLS support and onset of suspected $\operatorname{HIT}(6,19,24)$.

\section{Data extraction and statistical analysis}

Studies included in the analysis were retrospective studies, 
case series or case reports. Data on retrospective studies and case series with more than two patients were extracted for meta-regression analysis. While retrospective studies and case series provided study-level data on larger amount of patients, they were far less granular. Therefore, individual patient-level data from case reports and, when possible, case series were extracted for more granular descriptive statistics as well as Kaplan-Meier survival analysis.

The incidence of HIT amongst those who received ECLS was analyzed by performing a meta-regression analysis of proportions using study-level pooled data from the retrospective institutional studies. Baseline characteristics and demographics were reported based on the patient-level data using descriptive statistics [median and interquartile range (IQR) or percentages] with the pooled number of individual patients as the denominator. Continuous variables were compared using Wilcoxon rank-sum test, while categorical variables were analyzed using Chi-square test. Patientlevel survival and outcome data were also used to produce descriptive statistics and Kaplan-Meier analysis. Separate subgroup analysis was performed on patients with VAECMO and VV-ECMO, when possible. All data analysis and visualization were performed with $\mathrm{R}$ software, version 3.5.1 (R Foundation for Statistical Computing, Vienna, Austria). P values $<0.05$ were considered statistically significant.

\section{Results}

\section{Study characteristics}

Overall, 4,471 articles were identified in the literature search. Following application of the inclusion and exclusion criteria, 19 studies were included, of which five studies were singlecenter retrospective studies, two were case series, and twelve were case reports (Table 1). A PRISMA flow diagram depicting the overall search strategy is shown in Figure 1. A total of 309 patients from five retrospective studies as well as one case series were pooled to analyze the incidence of suspected and confirmed HIT in patients on ECLS. Whereas, 28 patients with confirmed HIT from twelve case reports, two case series, as well as two retrospective studies with individual patient data were pooled for a more granular univariate analysis and production of Kaplan-Meier survival curves. A manual search of references did not yield further studies.

\section{Patient characteristics}

Out of 309 patients identified in the retrospective studies and case series who underwent ECLS, $83 \%$ (95\% CI, 47-96) were suspected, and $17 \%$ (95\% CI, 5-48) were confirmed to have HIT (Table 2). It is important to note that there was significant heterogeneity in HIT incidence on ECLS $\left(\mathrm{I}^{2}=86 \%, \mathrm{P}<0.01\right)$.

Univariate analysis for the 28 patients who were identified to have confirmed HIT was subsequently performed. Baseline patient demographics for the patients with confirmed HIT are shown in Table 3. The median age at the time of ECLS treatment was 43.0 (IQR, 30.2-58.2) and $18(64.3 \%)$ were male. VA-ECMO was utilized in 15 patients $(53.6 \%)$, whereas VV-ECMO was used in 13 patients $(46.4 \%)$. The most common presentation was acute respiratory distress syndrome (32.1\%) followed by myocardial infarction $(21.4 \%)$. Detailed breakdown of indication for ECLS is presented in Table 3.

\section{ECLS-related characteristics}

ECLS-related characteristics are shown in Table 4. Patients supported on VV-ECMO tend to have shorter duration of support compared to those on VA-ECMO [VA-ECMO: 9 (IQR, 8-12) days vs. VV-ECMO: 20 (IQR, 11-28) days, $\mathrm{P}=0.081]$. ECLS cannulation was performed centrally in $13.3 \%$ of all patients, all of which were on VA-ECMO, whereas $86.7 \%$ were cannulated peripherally. Additional support such as intra-aortic balloon pump $(\mathrm{n}=2,7.1 \%)$, Impella $(n=1,3.6 \%)$ and hemodialysis $(n=4,14.3 \%)$ was required alongside ECLS. Concomitant surgeries were performed in 5 patients (17.9\%) while on ECLS support.

\section{HIT-related variables, diagnosis and treatment}

Overall, patients were on heparin for a median of 9 (IQR, 6-11) days [VA-ECMO: 8 (IQR, 6-9) days vs. VV-ECMO: 11 (IQR, 5-17) days, $\mathrm{P}=0.247$ ] and the median time to platelet nadir was 6 (IQR, 6-10) days [VA-ECMO: 6 (IQR, 6-6) days vs. VVECMO: 11 (IQR, 6-14) days, $\mathrm{P}=0.119$ ] (Table 5). Patients on VA-ECMO support had a significantly lower median platelet count pre-nadir [VA-ECMO: 100.5 (IQR, 88.0-135.2) per $\mu \mathrm{L}$ vs. VV-ECMO: 169.0 (IQR, 132.8-256.0) per $\mu \mathrm{L}, \mathrm{P}=0.035$ ] and median platelet count nadir [VA-ECMO: 26.0 (IQR, 20.0-33.5) per $\mu \mathrm{L} v s$. VV-ECMO: 45.0 (IQR, 38.0-60.0) per $\mu \mathrm{L}, \mathrm{P}=0.012]$. A positive $\mathrm{H}-\mathrm{PF} 4 \mathrm{Ab}$ ELISA test was observed in 25 patients (89.3\%), a positive SRA in 9 patients $(32.1 \%)$, and a positive HIPA in 6 patients (21.4\%).

Overall, 22 patients $(78.6 \%)$ were treated with argatroban, $4(14.3 \%)$ were treated with bivalirudin, and 


\begin{tabular}{|c|c|c|c|c|c|c|c|c|}
\hline First author & Year & Institution & $\begin{array}{l}\text { Study period } \\
\text { (years) }\end{array}$ & Type & $\begin{array}{l}\text { \# of patients } \\
\text { on ECLS }\end{array}$ & VA-ECMO & VV-ECMO & $\begin{array}{l}\text { \# of patients } \\
\text { with HIT }\end{array}$ \\
\hline Glick (12) & 2015 & $\begin{array}{l}\text { Columbia University Medical } \\
\text { Center, New York, NY, USA }\end{array}$ & 2011-2013 & Retrospective & 119 & 54 & 65 & 1 \\
\hline Sokolovic (6) & 2016 & $\begin{array}{l}\text { Medstar Washington Hospital } \\
\text { Center, Washington, DC, USA }\end{array}$ & 2009-2013 & Retrospective & 96 & 96 & 0 & 8 \\
\hline Laverdure (11) & 2016 & $\begin{array}{l}\text { Hôpital Marie Lannelongue, } \\
\text { Le Plessis Robinson, France }\end{array}$ & N/A & Retrospective & 73 & $\mathrm{~N} / \mathrm{A}$ & $\mathrm{N} / \mathrm{A}$ & 3 \\
\hline Beiderlinden (5) & 2007 & $\begin{array}{l}\text { Universität Duisburg-Essen, } \\
\text { Germany }\end{array}$ & 2005-2006 & Retrospective & 9 & 0 & 9 & 3 \\
\hline Hillebrand (7) & 2015 & $\begin{array}{l}\text { University Hospital of the } \\
\text { Westfaelische Wilhelms- } \\
\text { University Muenster, Germany }\end{array}$ & 2012-2013 & Retrospective & 7 & $\mathrm{~N} / \mathrm{A}$ & N/A & 6 \\
\hline Natt (8) & 2017 & $\begin{array}{l}\text { University of Arizona Medical } \\
\text { Center, Tucson, AZ, USA }\end{array}$ & $\mathrm{N} / \mathrm{A}$ & Case series & 5 & 0 & 5 & 3 \\
\hline Rougé (19) & 2017 & $\begin{array}{l}\text { Universite de Grenoble Alpes, } \\
\text { Grenoble, France }\end{array}$ & $\mathrm{N} / \mathrm{A}$ & Case series & 2 & 1 & 1 & 2 \\
\hline Chen (15) & 2017 & $\begin{array}{l}\text { University of Texas } \\
\text { Southwestern Medical Center, } \\
\text { Dallas, TX, USA }\end{array}$ & N/A & Case report & 1 & 1 & 0 & 1 \\
\hline Dager (25) & 2004 & $\begin{array}{l}\text { University of California Davis, } \\
\text { Davis, CA, USA }\end{array}$ & $\mathrm{N} / \mathrm{A}$ & Case report & 1 & 0 & 1 & 1 \\
\hline Dolch (24) & 2010 & $\begin{array}{l}\text { University Hospital } \\
\text { Grosshadern, Munich, } \\
\text { Germany }\end{array}$ & N/A & Case report & 1 & 0 & 1 & 1 \\
\hline Ito (16) & 2017 & $\begin{array}{l}\text { Tokyo Metropolitan Tama } \\
\text { Medical Center, Fuchu, Japan }\end{array}$ & $\mathrm{N} / \mathrm{A}$ & Case report & 1 & 1 & 0 & 1 \\
\hline Koster (21) & 2017 & $\begin{array}{l}\text { Ruhr-University Bochum, } \\
\text { Germany }\end{array}$ & N/A & Case report & 1 & 0 & 1 & 1 \\
\hline Koster (18) & 2007 & $\begin{array}{l}\text { Deutsches Herzzentrum } \\
\text { Berlin, Germany }\end{array}$ & N/A & Case report & 1 & 1 & 0 & 1 \\
\hline Phillips (9) & 2014 & $\begin{array}{l}\text { University of North Carolina, } \\
\text { Chapel Hill, NC, USA }\end{array}$ & N/A & Case report & 1 & 0 & 1 & 1 \\
\hline Ratzlaff (20) & 2016 & $\begin{array}{l}\text { Mayo Clinic Florida, } \\
\text { Jacksonville, FL, USA }\end{array}$ & N/A & Case report & 1 & 0 & 1 & 1 \\
\hline Gellatly (10) & 2014 & $\begin{array}{l}\text { Alfred Hospital, Melbourne, } \\
\text { Australia }\end{array}$ & N/A & Case report & 1 & 1 & 0 & 1 \\
\hline Sandoval (22) & 2018 & $\begin{array}{l}\text { ICCV Hospital Clinic, } \\
\text { Barcelona, Spain }\end{array}$ & N/A & Case report & 1 & 1 & 0 & 1 \\
\hline $\operatorname{Sin}(17)$ & 2017 & $\begin{array}{l}\text { Massachusetts General } \\
\text { Hospital, Boston, MA, USA }\end{array}$ & N/A & Case report & 1 & 0 & 1 & 1 \\
\hline Welp (23) & 2014 & $\begin{array}{l}\text { University Hospital Münster, } \\
\text { Germany }\end{array}$ & $\mathrm{N} / \mathrm{A}$ & Case report & 1 & 1 & 0 & 1 \\
\hline
\end{tabular}

ECLS, extracorporeal life support; ECMO, extracorporeal membrane oxygenation; HIT, heparin-induced thrombocytopenia; N/A, not applicable; VA, veno-arterial; VV, veno-venous. 


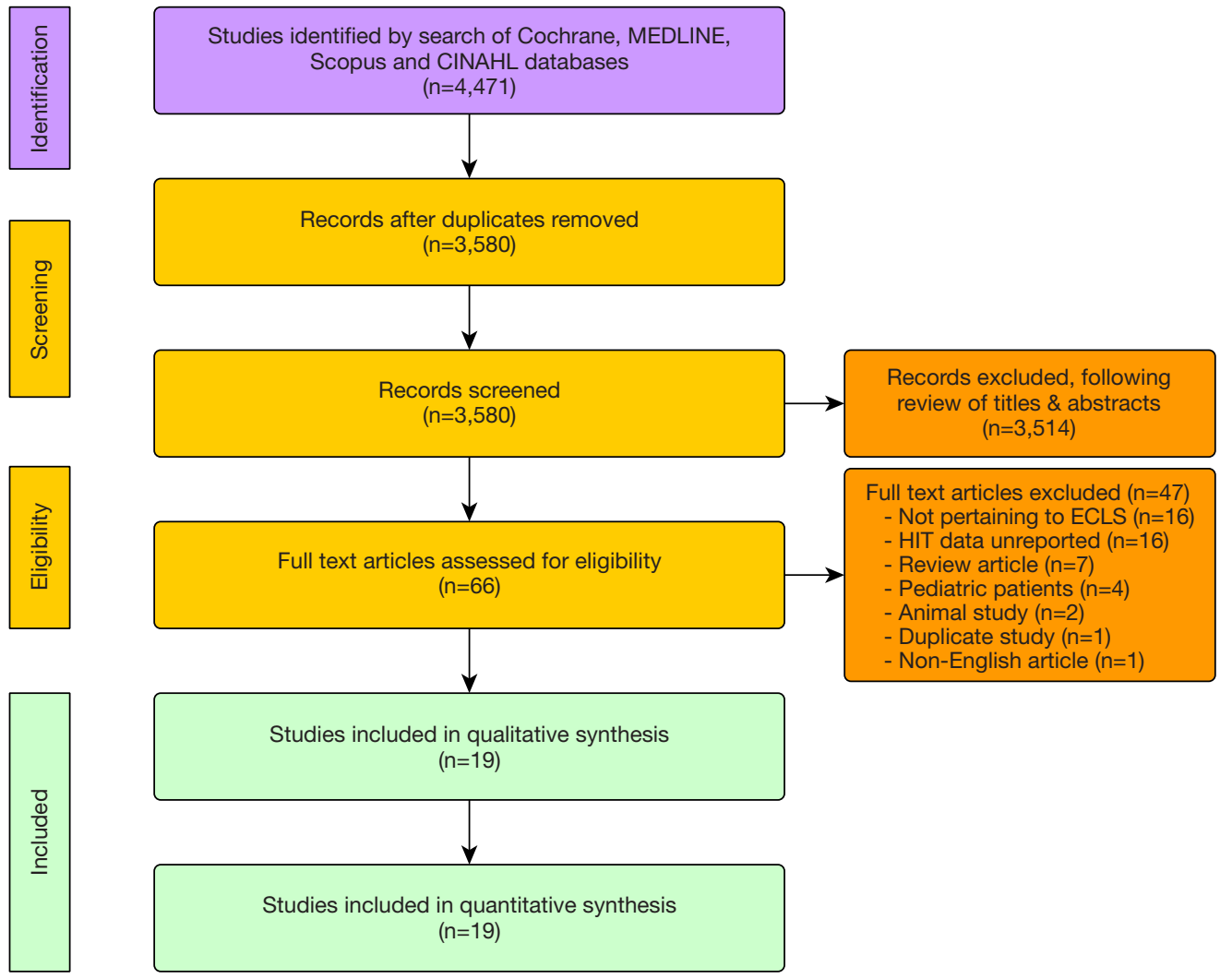

Figure 1 PRISMA schematic diagram of the search strategy. PRISMA, preferred reporting items for systematic reviews and meta-analysis. ECLS, extracorporeal life support; HIT, heparin-induced thrombocytopenia.

Table 2 Incidence of suspected heparin induced thrombocytopenia (HIT) and confirmed HIT in patients on extracorporeal membrane oxygenation (ECMO), pooled from the five retrospective studies and one case series

\begin{tabular}{lllll}
\hline Patients on ECMO & Pooled value $(\%)[95 \% \mathrm{Cl}]$ & No. of studies & No. of patients $(\mathrm{n} / \mathrm{N})$ & $187 / 309$ \\
\hline HIT suspected & $83[47-96]$ & 6 & $24 / 309$ \\
\hline HIT confirmed & $17[5-48]$ & 6 & $94^{*}$ \\
\hline${ }^{*}$, heterogeneity $\mathrm{P}<0.05$ (significant data heterogeneity present). & & & $86^{*}$ \\
\hline
\end{tabular}

$2(7.1 \%)$ were treated with lepirudin. Details regarding the medical treatment for HIT are illustrated in Table 6. In addition, platelet transfusion was provided to 5 patients (17.9\%) whereas 1 patient on VA-ECMO (3.6\%) underwent plasmapheresis. There were no significant differences amongst the various treatment options for patients on VAECMO as compared to VV-ECMO.

\section{Complications}

Complications experienced by patients on ECLS with HIT included 15 (53.6\%) patients with thrombosis [VA-ECMO, $\mathrm{n}=10(66.7 \%)$ vs. VV-ECMO, $\mathrm{n}=5(38.5 \%), \mathrm{P}=0.266]$ and $6(21.4 \%)$ patients with bleeding [VA-ECMO, $\mathrm{n}=2$ (13.3\%) vs. VV-ECMO, $\mathrm{n}=4$ (30.8\%), $\mathrm{P}=0.509]$. Arterial thromboembolism occurred in 8 patients $(28.6 \%)$, venous thromboembolism occurred in 6 patients $(21.4 \%)$, and ECLS circuit thrombosis in 6 patients (21.4\%). Patients supported on VA-ECMO were more likely to experience arterial thromboembolism as compared to VV-ECMO [VA$\mathrm{ECMO}, \mathrm{n}=8$ (53.3\%) vs. VV-ECMO, $\mathrm{n}=0$ (0\%), $\mathrm{P}=0.007]$ with venous thromboembolism incidence comparable 


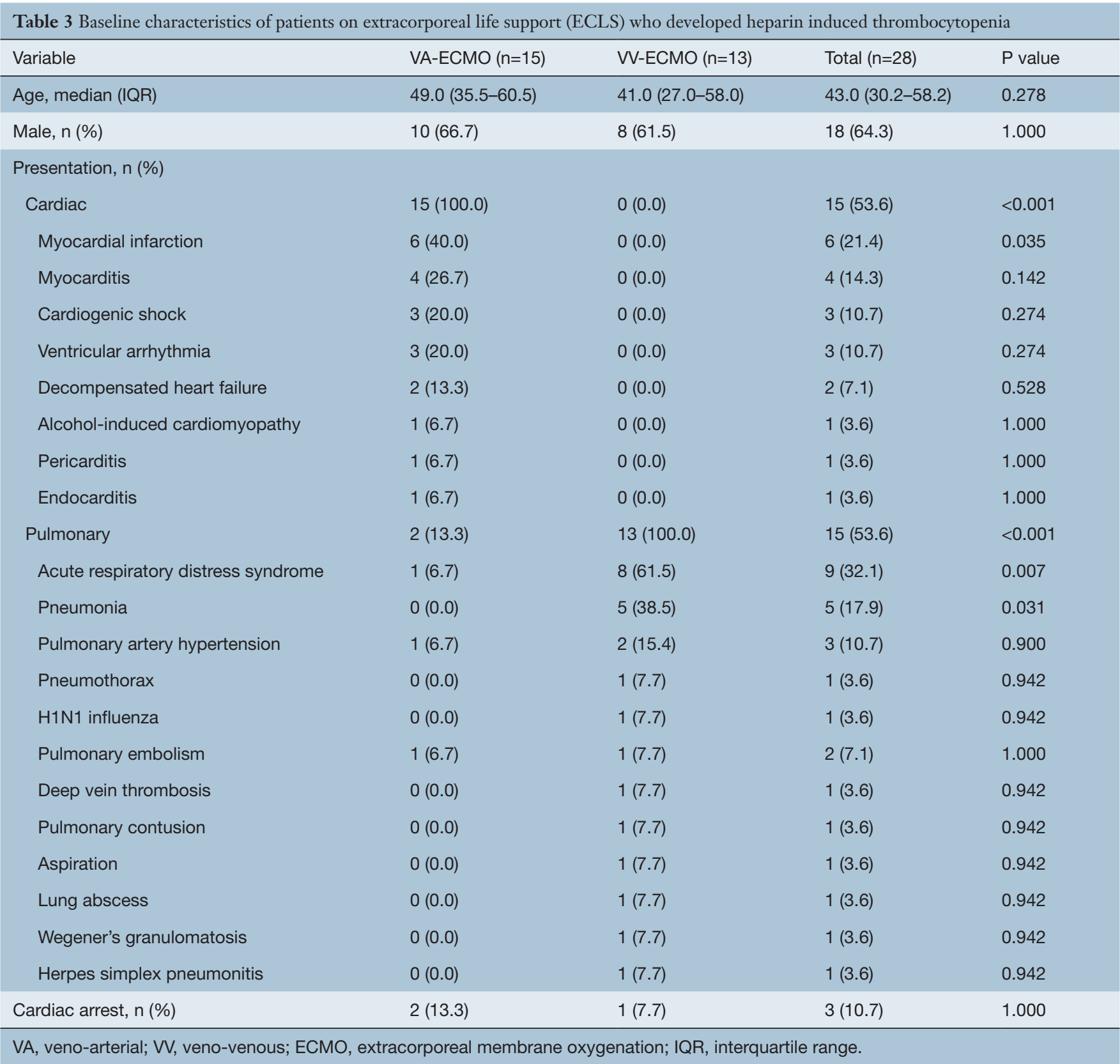

between the two groups [VA-ECMO, $\mathrm{n}=5(33.3 \%) v s$. VVECMO, $\mathrm{n}=1$ (7.7\%), $\mathrm{P}=0.235$ ] (Table 7). However, patients on VA-ECMO had a trend towards decreased likelihood of experiencing ECLS circuit oxygenator thromboembolism [VA-ECMO, $\mathrm{n}=0(0.0 \%)$ vs. VV-ECMO, $\mathrm{n}=4$ (30.8\%), $\mathrm{P}=0.075]$ and thromboembolism necessitating ECMO device or circuit exchange [VA-ECMO, $\mathrm{n}=2(13.3 \%) v s$. VV-ECMO, $\mathrm{n}=7$ (53.8\%), $\mathrm{P}=0.060]$.

\section{Outcomes and survival}

Median hospital length of stay was 42 (IQR, 28-70) days for all patients, with no significant difference between VA and VV-ECMO group [VA-ECMO, 51 (IQR, 35-61) days vs. VV-ECMO, 35 (IQR, 23-79) days, $\mathrm{P}=0.855]$. The median follow-up time from admission was 60 (IQR, 39-123) days, the follow up time from ECLS initiation was 96 (IQR, 
Table 4 Extracorporeal life support (ECLS) characteristics

\begin{tabular}{|c|c|c|c|c|}
\hline Variable & VA-ECMO (n=15) & VV-ECMO $(n=13)$ & Total $(n=28)$ & $P$ value \\
\hline Cannulation, $\mathrm{n}(\%)^{\star}$ & & & & 0.278 \\
\hline Central & $2(33.3)$ & $0(0.0)$ & $2(13.3)$ & \\
\hline Peripheral & $4(66.7)$ & $9(100.0)$ & $13(86.7)$ & \\
\hline Intra-aortic balloon pump & $2(13.3)$ & $0(0.0)$ & $2(7.1)$ & 0.528 \\
\hline Impella & $1(6.7)$ & $0(0.0)$ & $1(3.6)$ & 1.000 \\
\hline Hemodialysis & $0(0.0)$ & $4(30.8)$ & $4(14.3)$ & 0.075 \\
\hline Additional/concomitant surgeries, n (\%) & $3(20.0)$ & $2(15.4)$ & $5(17.9)$ & 1.000 \\
\hline BiVAD surgery & $1(6.7)$ & $0(0.0)$ & $1(3.6)$ & 1.000 \\
\hline Colectomy & $1(6.7)$ & $0(0.0)$ & $1(3.6)$ & 1.000 \\
\hline Hemothorax evacuation & $0(0.0)$ & $1(7.7)$ & $1(3.6)$ & 0.942 \\
\hline
\end{tabular}

VA, veno-arterial; VV, veno-venous; ECMO, extracorporeal membrane oxygenation; IQR, interquartile range; RVAD, right ventricular assist device; BiVAD, biventricular assist device. *, provided for six patients in the VA-ECMO group, and nine patients in the VV-ECMO group.

Table 5 Heparin-induced thrombocytopenia diagnosis and treatment in patients on VA as compared to VV-ECMO

\begin{tabular}{|c|c|c|c|c|}
\hline Variable & VA-ECMO $(n=15)$ & VV-ECMO $(n=13)$ & Total $(n=28)$ & $P$ value \\
\hline Positive serotonin release assay, $\mathrm{n}(\%)$ & $6(40.0)$ & $3(23.1)$ & $9(32.1)$ & 0.582 \\
\hline Positive heparin induced platelet aggregation, $\mathrm{n}(\%)$ & $2(13.3)$ & $4(30.8)$ & $6(21.4)$ & 0.509 \\
\hline Days on heparin, median [IQR] & 8 [6-9] & $11[5-17]$ & $9[6-11]$ & 0.247 \\
\hline Platelet count pre-nadir $(/ \mu \mathrm{L})$, median [IQR] & $100.5[88.0-135.2]$ & $169.0[132.8-256.0]$ & $131.0[90.0-190.5]$ & 0.035 \\
\hline Platelet count nadir $(/ \mu \mathrm{L})$, median [IQR] & $26.0[20.0-33.5]$ & $45.0[38.0-60.0]$ & $34.0[23.0-52.0]$ & 0.012 \\
\hline \multicolumn{5}{|l|}{ Treatment, n (\%) } \\
\hline Medication & & & & 0.981 \\
\hline Lepirudin & $1(6.7)$ & $1(7.7)$ & $2(7.1)$ & \\
\hline Platelet transfusion & $1(6.7)$ & $4(30.8)$ & 5 (17.9) & 0.244 \\
\hline Plasmapheresis & $1(6.7)$ & $0(0.0)$ & $1(3.6)$ & 1.000 \\
\hline
\end{tabular}

VA, veno-arterial; VV, veno-venous; ECMO, extracorporeal membrane oxygenation; H-PF4, heparin-platelet factor 4; ELISA, enzyme-linked immunosorbent assay; IQR, interquartile range. 
Table 6 Summary of the medical treatment of heparin induced thrombocytopenia (HIT) using direct thrombin inhibitors

\begin{tabular}{|c|c|c|c|c|c|c|}
\hline Medication & $\begin{array}{l}\text { Initial infusion dose } \\
(\mu \mathrm{g} / \mathrm{kg} / \mathrm{min})\end{array}$ & $\begin{array}{l}\text { Treatment duration } \\
\text { (days) }\end{array}$ & $\begin{array}{l}\text { Target ACT, lower } \\
\text { range (seconds) }\end{array}$ & $\begin{array}{l}\text { Target ACT, upper } \\
\text { range (seconds) }\end{array}$ & $\begin{array}{l}\text { Target aPT, lower } \\
\text { range (seconds) }\end{array}$ & $\begin{array}{l}\text { Target aPTT, upper } \\
\text { range (seconds) }\end{array}$ \\
\hline $\begin{array}{l}\text { Argatroban, } \\
\text { median [IQR] }\end{array}$ & $0.2[0.2-0.7]$ & 11 [8-36] & $165[163-168]^{\star \star}$ & $190[185-195]^{\star \star}$ & 50 [49-53] & 60 [60-60] \\
\hline $\begin{array}{l}\text { Hirudin derivatives } \\
\text { (all), median }[\mathrm{IQR}]\end{array}$ & $5.0[3.5-6.5]^{\star \star}$ & $4[3-5]^{\star \star}$ & 180 [165-190] & 220 [210-260] & $45[45-45]^{*}$ & $50[50-50]^{\star}$ \\
\hline $\begin{array}{l}\text { Lepirudin, median } \\
\text { [IQR] }\end{array}$ & $2.0[2.0-2.0]^{*}$ & $6[6-6]^{*}$ & $150[150-150]^{\star}$ & $200[200-200]^{\star}$ & $45[45-45]^{*}$ & $50[50-50]^{\star}$ \\
\hline
\end{tabular}

${ }^{*}$, based on a single case report; ${ }^{*}$, based on two case reports. IQR, interquartile range; ACT, activated clotting time; aPTT, activated partial thromboplastin time.

Table 7 Complications following heparin-induced thrombocytopenia in patients on extracorporeal life support (ECLS)

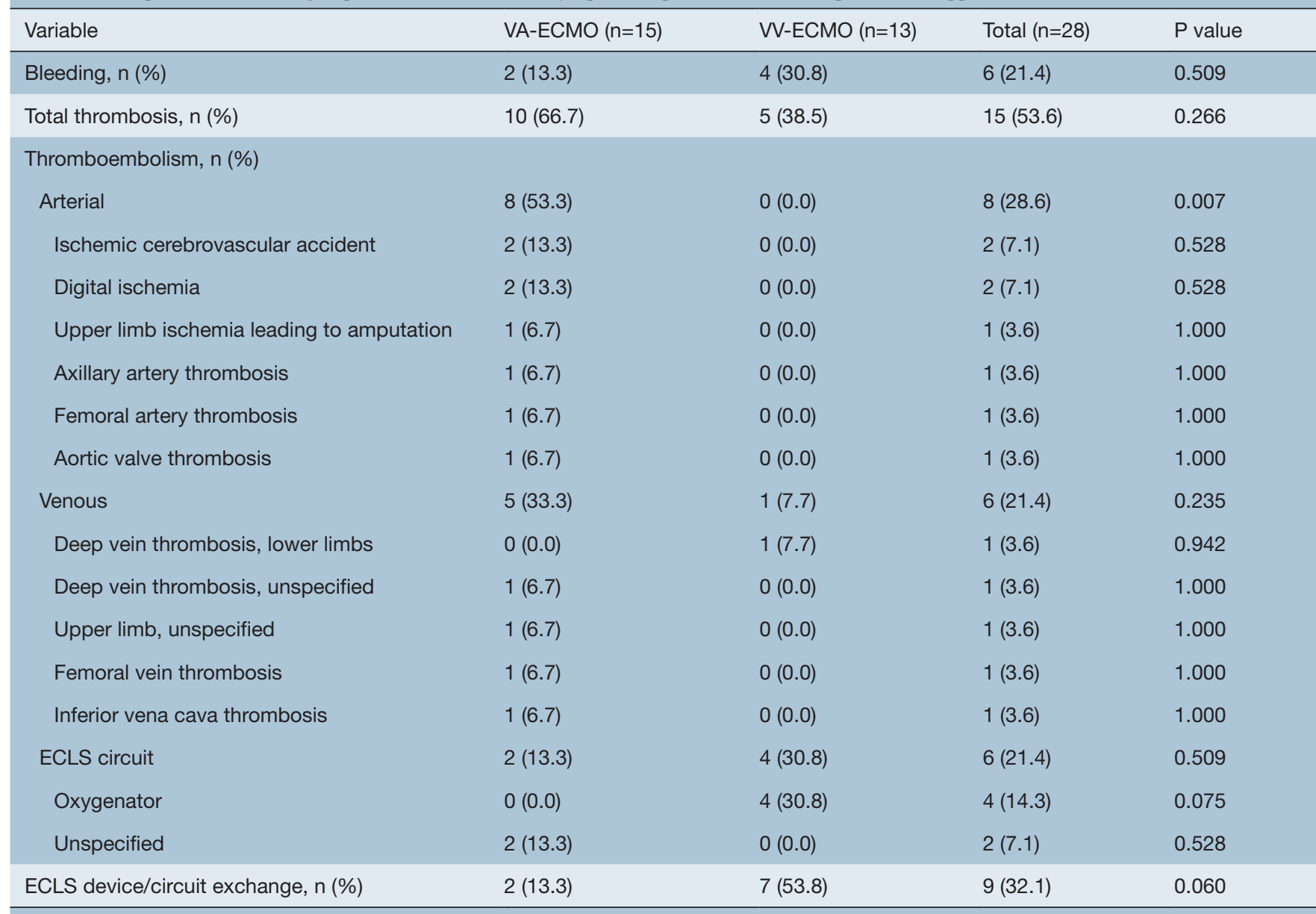

VA, veno-arterial; VV, veno-venous; ECMO, extracorporeal membrane oxygenation; ECLS, extracorporeal life support. 


\begin{tabular}{|c|c|c|c|c|}
\hline Hospital stay (days), median [IQR] & $51[35-61]$ & 35 [23-79] & $42[28-70]$ & 0.855 \\
\hline Follow up from admission (days), median [IQR] & $86[60-211]$ & 35 [23-79] & 60 [39-123] & 0.143 \\
\hline Follow up from ECLS (days), median [IQR] & 172 [96-253] & 54 [24-92] & $96[44-156]$ & 0.149 \\
\hline Overall survival, n (\%) & $8(53.3)$ & $8(61.5)$ & $16(57.1)$ & 0.956 \\
\hline Heart transplant, n (\%) & $1(6.7)$ & $0(0.0)$ & $1(3.6)$ & 1.000 \\
\hline Lung transplant, n (\%) & $0(0.0)$ & $2(15.4)$ & $2(7.1)$ & 0.400 \\
\hline \multicolumn{5}{|l|}{ Causes of death, n (\%) } \\
\hline Sepsis & $1(6.7)$ & $0(0.0)$ & $1(3.6)$ & 1.000 \\
\hline ARDS & $0(0.0)$ & $1(7.7)$ & $1(3.6)$ & 0.942 \\
\hline H1N1 influenza & $0(0.0)$ & $1(7.7)$ & $1(3.6)$ & 0.942 \\
\hline Pulmonary contusion & $0(0.0)$ & $1(7.7)$ & $1(3.6)$ & 0.942 \\
\hline Unspecified & $6(40.0)$ & $0(0.0)$ & $6(21.4)$ & 0.035 \\
\hline
\end{tabular}

44-156) days and the follow up time from platelet nadir was 88 (IQR, 25-111) days (Table 8).

A total of 16 patients (57.1\%) survived until the end of follow-up. Causes of death are shown in Table 8. One patient $(3.6 \%)$ supported on VA-ECMO underwent heart transplantation whereas 2 patients $(7.1 \%)$ supported on VV-ECMO underwent lung transplantation. Kaplan-Meier survival plots from time of admission (Figure 2) reveal no significant differences in survival in patients supported on VA-ECMO as compared to VV-ECMO.

\section{Discussion}

ECMO maintains cardiopulmonary support, independent of the lungs (VV-ECMO) and/or heart (VA-ECMO), providing a temporary bridge to recovery, transplantation, or long-term mechanical circulatory support after acute pulmonary and/or cardiac failure (1). The interaction of the nonbiological surface of an extracorporeal circuit and blood leads to altered coagulation (26). The Extracorporeal Life Support Organization (ELSO) guidelines suggest a continuous infusion of unfractionated heparin during ECLS to achieve anticoagulation and use of heparin-coated circuits $(27,28)$. HIT is a severe adverse event with thromboembolic complications secondary to exposure to heparin (29). There is paucity in the report of incidence as well as guidelines to aid in the monitoring, diagnosis and management of HIT in patients on ECLS.

In this systematic review and meta-analysis of HIT in ECLS, we demonstrate that of 309 pooled patients, 17\% were confirmed to have HIT. Of the 28 patients with confirmed HIT on ECLS, $53.6 \%$ and $46.4 \%$ had undergone VA-ECMO and VV-ECMO, respectively. Patients on VA-ECMO had a lower median platelet count nadir and were more likely to experience arterial thromboembolism, though had a trend towards decreased likelihood of experiencing ECLS circuit oxygenator thromboembolism and thromboembolism necessitating ECLS device or circuit exchange. It is possible that differences in heparin dosing and partial thromboplastin time targets between patients on VA-ECMO and VV-ECMO contribute to the differences observed. Kaplan-Meier survival plots from time of admission reveal no significant differences in survival in patients supported on VA-ECMO as compared to VV- 

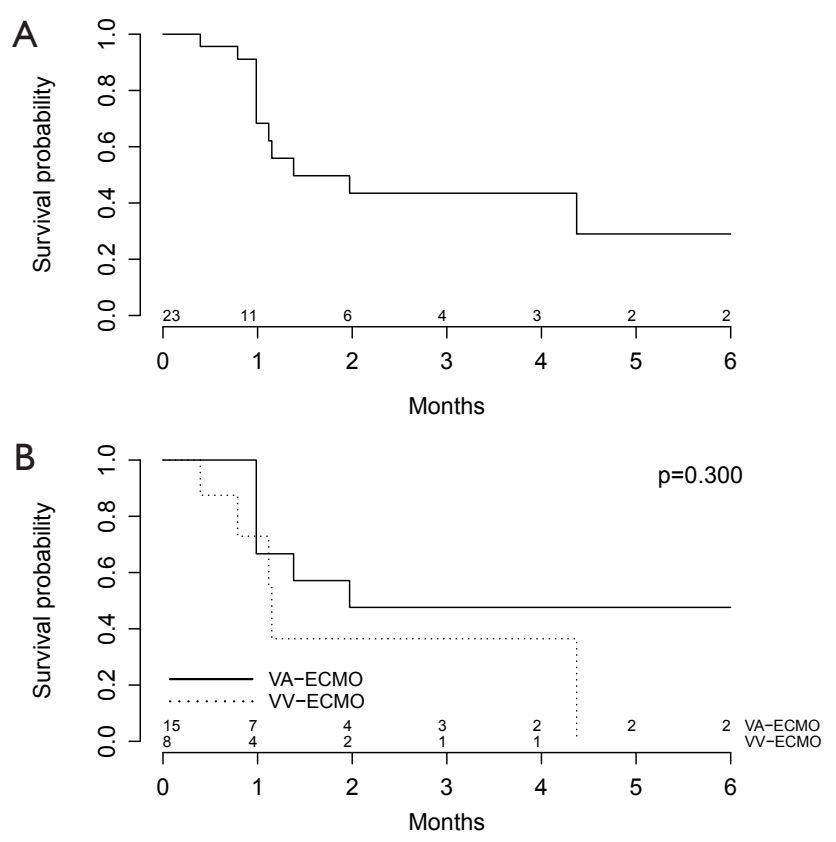

Figure 2 Estimated survival of patients with confirmed HIT post-ECLS during follow-up after time of admission, (A) in all patients and (B) stratified by VA-ECMO and VV-ECMO groups. HIT, heparin-induced thrombocytopenia; ECLS, extracorporeal life support; VA, veno-arterial; VV, veno-venous; ECMO, extracorporeal membrane oxygenation.

\section{ECMO.}

The diagnosis of HIT is based on both clinical suspicion and pathologic confirmation (12). Clinical suspicion of HIT typically occurs with declining platelet counts in the setting of active heparin use. However, thrombocytopenia in critically ill patients is often multifactorial and may be due to various inciting events making it difficult to distinguish HIT from non-HIT thrombocytopenia in patients on ECLS (12). Common causes of thrombocytopenia that mimic the presentation of HIT $(28,30)$ include circuit related effects of mechanical circulatory support causing platelet activation and aggregation (26,31), sepsis, medications, surgery, bleeding, hemodilution, intravascular devices and blood transfusions (11).

Patients on ECLS with HIT have much higher odds for thromboembolism-independent of time spent on ECLS (6). As such, recognition of HIT in ECLS is critical, as it requires an urgent change in therapy. The incidence of HIT in our study is higher than previously reported in patients after cardiothoracic surgery $(0.47 \%)$ (32) or during ECLS $(4.1 \%$ and $0.8 \%)(11,12)$ which emphasizes the challenges of diagnosing HIT in patients on ECLS. As shown in our study, there is limited accord between $\mathrm{H}-\mathrm{PF} 4$ ELISA screening results and confirmed HIT cases for patients on ECLS (3). Furthermore, HIT may still be present in patients on ECLS despite negative testing $(5,33)$. According to our analysis, the SRA was positive only in $32 \%$ of clinically confirmed HIT, which may indicate that the test may not be specific enough. In fact, the IgGspecific ELISA has been shown to have superior specificity when compared to the SRA since among IgG, IgA, and IgM antibodies that can be detected against the H-PF4 complex, only the IgG antibody class can bind to the Fc receptor and cause subsequent platelet activation, resulting in the prothrombotic state (34).

We demonstrate that patients supported on VA-ECMO were more likely to have a lower platelet count nadir $(\mathrm{P}=0.012)$ and pre-nadir $(\mathrm{P}=0.035)$ as compared to those supported on VV-ECMO. In terms of complications, as VAECMO drains venous blood, oxygenates and subsequently inputs into the arterial system (35), it is not surprising that patients with HIT on VA-ECMO were more likely to experience arterial thromboembolism as compared to those on VV-ECMO $(\mathrm{P}=0.007)$. It is possible that decreased heparin dosing and partial thromboplastin time targets in patients on VV-ECMO contribute to these differences observed. Hence, with the same logic, patients on VVECMO would have a lower incidence of HIT, higher platelet count nadir and resulting increased incidence of ECMO circuit oxygenator thromboembolism $(\mathrm{P}=0.075)$ and thromboembolism necessitating ECLS device or circuit exchange $(\mathrm{P}=0.060)$. Unfortunately, we were unable to differentiate the incidence of these complications in patients on different ECMO modalities.

Other potential explanations for the differences observed between patients on VA-ECMO as compared to $\mathrm{VV}$ ECMO include that patients on VA-ECMO are a different population with different etiology of cardiorespiratory failure and concomitant illnesses that may predispose them to have lower platelet count pre-nadir which continues to a lower platelet count nadir. It is also plausible that the hemodynamic properties of VA-ECMO with different perfusion rates and pressures in the venous and arterial system lead to higher shear and consequent platelet activation and aggregation $(1,26,31,35)$.

According to data from the annual international ELSO Registry Reports (36), of 16,337 patients that received ECLS for pulmonary support, 59\% survived to discharge; whereas of 15,942 patients that received ECLS for cardiac 
support, $42 \%$ survived to discharge. The similar survival amongst patients supported on VA-ECMO as compared to VV-ECMO with HIT may be due to the low number of patients included in the study and resulting lack of power in discerning significant differences (36).

Rapid and accurate diagnosis is paramount to avoid the perils of misdiagnosis of HIT in patients on ECLS. Consensus on heparin monitoring and therapeutic targets, thorough inspection of the ECLS oxygenator (6), monitoring pressures throughout the circuit (6) and evaluation of system efficiency (lower $\mathrm{PaO}_{2}$ and higher $\mathrm{PaCO}_{2}$ ) (6) as well as systematic use of the 4Ts (37) and HIT Expert Probability Score (38) may help guide the decision to test for HIT, though, these scoring systems have not been validated in patients receiving ECLS (37-39). Without a validated pretest probability clinical score, maintaining a high index of suspicion for HIT (8) and serosurveillance in a defined high-risk patient on ECLS may be needed (6).

Limited experience with alternative anticoagulants and various doses on ECLS include fondaparinux (10), danaparoid (40), lepirudin $(10,25,41)$, argatroban $(5,7,19,24,42)$, and bivalirudin (18) with variable success. The 2012 American College of Chest Physicians HIT guidelines did not provide guidance for patients on ECLS support. However, they recommend bivalirudin for patients with acute HIT who require urgent cardiac surgery over other non-heparin anticoagulants and over heparin plus antiplatelet agents (grade 2C) and bivalirudin (grade 2B) or argatroban (grade 2C) for patients with HIT requiring acute percutaneous coronary interventions (4). Not specific for patients on ECLS, the American Society of Apheresis has maintained a class $2 \mathrm{C}$ recommendation for plasmapheresis as a therapeutic option for HIT (43). Although there was conflicting initial data, platelet transfusion seems safe in patients with HIT, but transfusion is only recommended in patients with clinical indications of bleeding or pending invasive procedures (4).

In light of ongoing uncertainty in anticoagulation strategy and anticoagulation monitoring in patients on ECLS, a large randomized controlled trial with the goal of defining the safest anticoagulation practice is currently in progress in Australia (ACTRN12613001324707). This study is being performed in patients without an additional indication for therapeutic anticoagulation, by comparing a standard systemic heparin anticoagulation strategy (aPTT target 50-70 seconds) and lower-dose heparin (12,000 units/24 h-adjusted for body weight and aiming for an aPTT b 45 seconds). Additionally, heparin alternatives should be subjected to clinical trials in these highrisk patients as use of a non-heparin anticoagulant may eliminate the clinical consequences of HIT.

\section{Limitations}

This meta-analysis has several key limitations and must be interpreted with care. Differences exist in individual patient comorbidities, etiology of cardiorespiratory failure, heparin formulations, ECLS circuit components and flow hemodynamics. An additional drawback is the retrospective, observational nature of the included studies and the lack of protocol for HIT screening, which was subject to individual clinician judgment. It is plausible that some patients not tested could have had HIT. Furthermore, due to the lack of granularity and availability of data, we were unable to assess whether there were differences in heparin exposure prior to ECLS as well as heparin dosing and partial thromboplastin time targets for all study patients, which would be a risk factor for HIT and thrombocytopenia, regardless of ECLS modality. This in turn led to the lack of statistical power in distinguishing the potential differences in the incidence of HIT between different ECLS modalities, as well as in complications such as oxygenator thrombosis and ECLS device exchange. We acknowledge that this heterogeneity in the study population and institutional protocol for anticoagulation on ECLS, HIT assessment, diagnosis and treatment is a fundamental limitation that cannot be addressed due to inability to extract sufficient detail from the pooled data. Publication bias as well as the small number of patients limits the statistical power of the analysis. It has to be also noted that, due to the nature of the data, KaplanMeier survival plots reflect the starting point from the time of admission and not from the diagnosis of HIT. These limitations restrict the broader applicability of the results presented in this study.

\section{Conclusions}

Patients who develop HIT on VA-ECMO are more likely to experience more severe thrombocytopenia and arterial thromboembolism than those on VV-ECMO; though, they had a trend towards decreased likelihood to have ECLS circuit oxygenator thromboembolism and thromboembolism necessitating ECLS device or circuit exchange. Further research in this area and development of standardized protocols for the monitoring, diagnosis and management of HIT in patients on ECLS support are warranted. 


\section{Acknowledgements}

None.

\section{Footnote}

Conflicts of Interest: The authors have no conflicts of interest to declare.

\section{References}

1. Murphy DA, Hockings LE, Andrews RK, et al. Extracorporeal membrane oxygenation-hemostatic complications. Transfus Med Rev 2015;29:90-101.

2. Lafç G, Budak AB, Yener AÜ, et al. Use of extracorporeal membrane oxygenation in adults. Heart Lung Circ 2014;23:10-23.

3. Cuker A. Clinical and laboratory diagnosis of heparininduced thrombocytopenia: an integrated approach. Semin Thromb Hemost 2014;40:106-14.

4. Linkins LA, Dans AL, Moores LK, et al. Treatment and prevention of heparin-induced thrombocytopenia: Antithrombotic Therapy and Prevention of Thrombosis, 9th ed: American College of Chest Physicians Evidence-Based Clinical Practice Guidelines. Chest 2012;141:e495S-530S.

5. Beiderlinden $M$, Treschan T, Görlinger K, et al. Argatroban in extracorporeal membrane oxygenation. Artif Organs 2007;31:461-5.

6. Sokolovic M, Pratt AK, Vukicevic V, et al. Platelet Count Trends and Prevalence of Heparin-Induced Thrombocytopenia in a Cohort of Extracorporeal Membrane Oxygenator Patients. Crit Care Med 2016;44:e1031-7.

7. Hillebrand J, Sindermann J, Schmidt C, et al. Implantation of left ventricular assist devices under extracorporeal life support in patients with heparin-induced thrombocytopenia. J Artif Organs 2015;18:291-9.

8. Natt B, Hypes C, Basken R, et al. Suspected HeparinInduced Thrombocytopenia in Patients Receiving Extracorporeal Membrane Oxygenation. J Extra Corpor Technol 2017;49:54-8.

9. Phillips MR, Khoury AI, Ashton RF, et al. The dosing and monitoring of argatroban for heparin-induced thrombocytopenia during extracorporeal membrane oxygenation: a word of caution. Anaesth Intensive Care 2014;42:97-8.

10. Gellatly RM, Leet A, Brown KE. Fondaparinux:
An effective bridging strategy in heparin-induced thrombocytopenia and mechanical circulatory support. J Heart Lung Transplant 2014;33:118.

11. Laverdure F, Louvain-Quintard V, Kortchinsky T, et al. PF4-heparin antibodies during ECMO: incidence, course, and outcomes. Intensive Care Med 2016;42:1082-3.

12. Glick D, Dzierba AL, Abrams D, et al. Clinically suspected heparin-induced thrombocytopenia during extracorporeal membrane oxygenation. J Crit Care 2015;30:1190-4.

13. Warkentin TE. HIT paradigms and paradoxes. J Thromb Haemost 2011;9:105-17.

14. Warkentin TE. How I diagnose and manage HIT. Hematology Am Soc Hematol Educ Program 2011;2011:143-9.

15. Chen E, Clarke N, Huffman L, et al. Transplantation in a patient on extracorporeal membrane oxygenation with infective endocarditis, pericarditis and heparin-induced thrombocytopenia. Interact Cardiovasc Thorac Surg 2017;24:462-3.

16. Ito K, Isogai T, Takada M, et al. Bilateral Arm Gangrene Associated With Heparin-Induced Thrombocytopenia After Extracorporeal Cardiopulmonary Resuscitation. Circ J 2017;82:293-4.

17. Sin JH, Lopez ND. Argatroban for Heparin-Induced Thrombocytopenia during Venovenous Extracorporeal Membrane Oxygenation with Continuous Venovenous Hemofiltration. J Extra Corpor Technol 2017;49:115-20.

18. Koster A, Weng Y, Böttcher W, et al. Successful Use of Bivalirudin as Anticoagulant for ECMO in a Patient With Acute HIT. Ann Thorac Surg 2007;83:1865-7.

19. Rougé A, Pelen F, Durand M, et al. Argatroban for an alternative anticoagulant in HIT during ECMO.J Intensive Care 2017;5:39.

20. Ratzlaff RA, Ripoll JG, Kassab LL, et al. Acute oxygenator failure: A new presentation of heparin-induced thrombocytopenia in a patient undergoing venovenous extracorporeal membrane oxygenation support. BMJ Case Rep 2016;2016. doi: 10.1136/bcr-2016-218179.

21. Koster A, Niedermeyer J, Gummert J, et al. Low dose bivalirudin anticoagulation for lung transplantation with extracorporeal membrane oxygenation in a patient with acute heparin-induced thrombocytopenia. Eur J Cardiothorac Surg 2017;51:1009-11.

22. Sandoval E, Ascaso M, Quintana E, et al. An infrequent complication of a not so infrequent disease. Hit by HIT. Asian Cardiovasc Thorac Ann 2018. [Epub ahead of print].

23. Welp H, Ellger B, Scherer M, et al. Heparin-induced thrombocytopenia during extracorporeal membrane 
oxygenation. J Cardiothorac Vasc Anesth 2014;28:342-4.

24. Dolch ME, Frey L, Hatz R, et al. Extracorporeal membrane oxygenation bridging to lung transplant complicated by heparin-induced thrombocytopenia. Exp Clin Transplant 2010;8:329-32.

25. Dager WE, Gosselin RC, Yoshikawa R, et al. Lepirudin in Heparin-Induced Thrombocytopenia and Extracorporeal Membranous Oxygenation. Ann Pharmacother 2004;38:598-601.

26. Warkentin TE, Greinacher A, Koster A. Heparin-induced thrombocytopenia in patients with ventricular assist devices: are new prevention strategies required? Ann Thorac Surg 2009;87:1633-40.

27. Esper SA, Levy JH, Waters JH, et al. Extracorporeal membrane oxygenation in the adult: a review of anticoagulation monitoring and transfusion. Anesth Analg 2014;118:731-43.

28. ELSO anticoagulation guidelines 2014;1-17. Available online: https://www.elso.org/Portals/0/Files/ elsoanticoagulationguideline8-2014-table-contents.pdf

29. Greinacher A. Heparin-Induced Thrombocytopenia. N Engl J Med 2015;373:1883-4.

30. Dewald O, Fischlein T, Vetter HO, et al. Platelet morphology in patients with mechanical circulatory support. Eur J Cardiothorac Surg 1997;12:634-41.

31. Schenk S, El-Banayosy A, Prohaska W, et al. Heparininduced thrombocytopenia in patients receiving mechanical circulatory support. J Thorac Cardiovasc Surg 2006;131:1373-81.e4.

32. Trehel-Tursis V, Louvain-Quintard V, Zarrouki Y, et al. Clinical and biologic features of patients suspected or confirmed to have heparin-induced thrombocytopenia in a cardiothoracic surgical ICU. Chest 2012;142:837-44.

33. Chan M, Malynn E, Shaz B, et al. Utility of consecutive repeat HIT ELISA testing for heparin-induced thrombocytopenia. Am J Hematol 2008;83:212-7.

34. Husseinzadeh HD, Gimotty PA, Pishko AM, et al. Diagnostic accuracy of IgG-specific versus polyspecific enzyme-linked immunoassays in heparin-induced

Cite this article as: Choi JH, Luc JG, Weber MP, Reddy HG, Maynes EJ, Deb AK, Samuels LE, Morris RJ, Massey HT, Loforte A, Tchantchaleishvili V. Heparin-induced thrombocytopenia during extracorporeal life support: incidence, management and outcomes. Ann Cardiothorac Surg 2019;8(1):19-31. doi: 10.21037/acs.2018.12.02 thrombocytopenia: a systematic review and meta-analysis. J Thromb Haemost 2017;15:1203-12.

35. Makdisi G, Wang IW. Extra Corporeal Membrane Oxygenation (ECMO) review of a lifesaving technology. J Thorac Dis 2015;7:E166-76.

36. Extracorporeal Life Support Registry Report. Available online: https://www.elso.org/Portals/0/Files/ Reports/2018_July/International\%20Summary\%20 July\%20First\%202018.pdf

37. Lo GK, Juhl D, Warkentin TE, et al. Evaluation of pretest clinical score (4 T's) for the diagnosis of heparin-induced thrombocytopenia in two clinical settings. J Thromb Haemost 2006;4:759-65.

38. Cuker A, Arepally G, Crowther MA, et al. The HIT Expert Probability (HEP) Score: a novel pre-test probability model for heparin-induced thrombocytopenia based on broad expert opinion. J Thromb Haemost 2010;8:2642-50.

39. Cuker A, Gimotty PA, Crowther MA, et al. Predictive value of the 4Ts scoring system for heparin-induced thrombocytopenia: a systematic review and meta-analysis. Blood 2012;120:4160-7.

40. Bauer C, Vichova Z, Ffrench P, et al. Extracorporeal membrane oxygenation with danaparoid sodium after massive pulmonary embolism. Anesth Analg 2008;106:1101-3, table of contents.

41. Balasubramanian SK, Tiruvoipati R, Chatterjee S, et al. Extracorporeal membrane oxygenation with lepirudin anticoagulation for Wegener's granulomatosis with heparin-induced thrombocytopenia. ASAIO J 2005;51:477-9.

42. Cornell T, Wyrick P, Fleming G, et al. A case series describing the use of argatroban in patients on extracorporeal circulation. ASAIO J 2007;53:460-3.

43. Schwartz J, Padmanabhan A, Aqui N, et al. Guidelines on the Use of Therapeutic Apheresis in Clinical PracticeEvidence-Based Approach from the Writing Committee of the American Society for Apheresis: The Seventh Special Issue. J Clin Apher 2016;31:149-62. 